\title{
A NON COOPERAPIVE GAME IN A DISTRIBUTED PARAMETER SYSTEM
}

\author{
I. F. YVON \\ IRIA - IABORIA \\ 73 - ROCQUENCOURT (FRANCE)
}

\section{ABSTRACT :}

This paper is devoted to study a class of differential games for distributed parameter systems. Essentially we study a Nash equilibrium point for a system governed by a parabolic equation. A method based on the SCARP-IIANSEN [1] algorlthm for -solution of non-cooperative games is given.

\section{I - INTRODUCTION}

The genergl formulation of non-cooperative games is the following .

(1.1) $\left\{\begin{array}{l}\text { Let } u_{i} \quad i=1,2, \ldots, n \text { be a family of Banach spaces } \\ \text { and } J_{i} i=1,2, \ldots, n \text { a family of functionals, each of } \\ \text { them being defined on } u=\prod_{i=1}^{\pi} u_{i} \text {. Then we have the following : }\end{array}\right.$

\section{Definition}

Let $u_{\mathrm{d} d}$ be a subset of $u$, a point $u \in u_{\mathrm{ad}}$ is a Nash equilibrium point if it satis-

(1.2) $\left\{\begin{array}{l}J_{i}\left(u_{1}, \ldots, u_{i-1}, u_{i}, u_{i+1}, \ldots, u_{n}\right) \leq J_{i}\left(u_{1}, \ldots, u_{i-1}, v_{i}, u_{i+1}, \ldots, u_{n}\right) \\ \text { for all } v_{i} \text { such that } \\ \left(u_{1}, \ldots, v_{i}, \ldots, u_{n}\right) \in u_{a d}\end{array}\right.$

This definition follows the paper of Nash [1]. The main result is then,

Theorem 1.1 ROSEN [1]

We assume that

(1.3) $u_{a d}$ is a convex compact subset of $u$

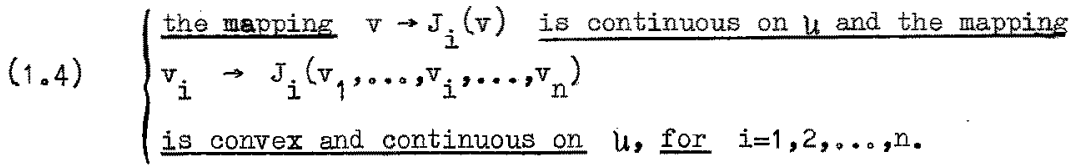

Then there exists at least an equilibrium noint on $u_{a d}$.

In order to be able to reat the infinite dimensionnel case we have the following extension of the result of ROSEN. 


\section{Theorem 1.2}

(1.5) If $u_{i}$ is a reflexive Banach space for $i=1,2, \ldots, n$ and $u_{a d}$ is a closed bouncontinuity in the weak topology of $u$.

\section{Remark 1.1}

Uniqueness of the solution requires more specific (and unfortuaately restrictive) hypothesis. The following assumption is as extension of monotonicity property :

If $J_{i}$ is Gateaux-differentiable with respect to $i t s i^{\text {th }}$ component and verifies the following property :

(1.6) $\left\{\begin{array}{l}\text { There exists a } \lambda_{1}, \ldots, \lambda_{n} \quad \lambda_{i} \geq 0 \text { such that } \\ \sum_{i=I}^{n} \lambda_{i}\left(\frac{\partial J_{i}}{\partial u_{i}}(u)-\frac{\partial J_{i}}{\partial u_{i}}(v), u_{i}-v_{i}\right)_{i} \geq 0\end{array}\right.$ where (,) denote the duality pairing between $V_{i}$ and $V_{i}^{\prime}$, then there exists a unique
equil.point.

\section{II - A CLASS OF DIFEERENTIAL GAMES}

Now we can state our game in the frame of optimal control theory for linear parabolic systems (cf. LIONS [1])。

Let $V$ and $H$ be two Hilbert spaces with $V$ dense in $H$ with continuous injection so that we have

$$
\mathrm{V} \subset \mathrm{H}=\mathrm{H}^{*} \subset \mathrm{V}^{\prime} \text { 。 }
$$

In addition we consider a family of operator

$$
A(t) \in \mathcal{L}\left(v_{;} \mathrm{V}^{\prime}\right) \quad t \in[0, \mathrm{~T}]
$$

and

$$
\left\{B_{i} \in \mathcal{L}\left(u_{i} ; L^{2}\left(0, T ; V^{\prime}\right)\right\}_{i=1,2, \ldots, n} .\right.
$$

If $f$ is given in $L^{2}\left(0, T ; V^{\prime}\right)$ et $y_{0} \in H$ we may consider the parabolic system

$$
\left\{\begin{array}{l}
\left.\frac{d y}{d t}+A(t) y=f+\sum_{i=1}^{n} B_{i} v_{i} \quad \text { sur } \quad\right] 0, T[ \\
y(0)=y_{0}
\end{array}\right.
$$

The equation admits a unique solution $y(v)$ depending continuously on $\nabla$ under assumption of "measurability" of $A(t)$ and coercitity of $A(t)$ :

$$
\left(\mathrm{A}(t)_{\varphi, \varphi}\right)_{V^{\prime} \mathrm{V}}+\lambda|\varphi|_{\mathrm{H}}^{2} \geq \alpha\|\varphi\|_{\mathrm{V}}^{2} \quad \forall \varphi \in V .
$$

Observation is given by a family of criteria

(2.2) $\quad\left\{\begin{array}{l}J_{i}(v)=\psi_{i}(y(v))+\theta_{i}(v) \text { where } \psi_{i} \text { is convex continuous in } y \text { and } \\ \theta_{i} \text { is convex continuous with respect to } v_{i}, \text { for } i=1,2, \ldots, n \\ \text { continuous with aspect to } v .\end{array}\right.$ 
If we assume that $u_{a d}$ is convex compact we can apply directly Theorem 1.1. If $u_{\text {ad }}$ is compact only for the weak topology of $u$ (eg。 $u_{\text {ad }}$ is a bounded closed convex subset of $u$ ) then we need a further assumption :

$$
\left\{\begin{array}{c}
v \rightarrow \psi(y(v))+\theta_{i}(v) \\
\text { is continuous for the weak topology of } u ;
\end{array}\right.
$$

An example of this situation is the following :

The linear-quadratic problem with distributed observation

We know (cf. LIONS [1]) that the solution $y(v)$ of $(2.1)$ belongs to $W(0, T)$ where

$$
W(0, T)=\left\{\varphi \in L^{2}(0, T ; V) \mid \frac{d \varphi}{d t} \in L^{2}\left(0, T ; V^{T}\right)\right\}
$$

which is an Hilbert space with the norm

(2.5) $\quad\|\varphi\|_{W}^{2}=\int_{0}^{T}\left\{\|\varphi(t)\|_{T}^{2}+\left\|\frac{d \varphi}{d t}(t)\right\|_{V^{2}}^{2}\right\} d t$.

(2.6) $\left\{\begin{array}{l}\text { Furthermore if the injection of } V \text { into } H \text { is compact then "the injection of } \\ W \text { in } \mathrm{L}^{2}(0, \mathrm{~T} ; \mathrm{H}) \text { is compact". }\end{array}\right.$

Hence if we consider the family of functional

$$
J_{i}(v)=\int_{0}^{T}\left\|y(v)-z_{d_{i}}\right\|_{\mathrm{H}}^{2} \bar{\alpha} t
$$

The assumption $(1.6)$ is verified; then we have :

Theorem 2.1

The linear-quadratic game given by (2.1) and (2.2) admits at least an equilibrium point in the bounded closed convex subset $u_{a d}$ of $u$, under assumption $(2.6)$.

Remark 2.1

Another approach for existence of a Nash point is, in the case of uncoupled contraints

$$
u_{a d}=\pi_{i=1}^{n} u_{a d, i} \quad u_{a d, i} \subset u_{i}
$$

is the theory of variational inequalities (cf. BENSOUSSAN [1], BENSOUSSAN-LIONSTEMAM [1]) and the compactness argument of Theorem 2.1 corresponis to a pseudomonotonicity property (in terminology of LIONS [2]) of the operator a defined by

$$
(a(u), v)=\Sigma\left(\frac{\partial J_{i}}{\partial v_{i}}\left(u_{i}\right), v_{i}\right) \text {. }
$$

\section{III - AN ALGORITEM FOR SOLUTION OF THE GAME}

In this section we give an example of a feasible algorithin (for solution of the game) based upon a method of SCARF [1].

If we consider the function 


$$
z(u, v)=\sum_{i=1}^{n} J_{i}\left(u_{1}, \ldots, u_{i-1}, v_{i}, u_{i+1}, \ldots, u_{n}\right)
$$

it is easy to show that the equilibrium point $(1)_{\text {is a fixed point for the mapping }}$

$$
u \rightarrow \Gamma(u)=\left\{v \in u_{a d} \mid z(u, v) \leq z(u, w) \quad \forall w \in u_{a d}\right\}
$$

Let us note that the mapping (3.2) is a point-to-point mapping in the case of strict convexity of $J_{i}$ for $i=1,2, \ldots, n$.

It follows from this remark that the search of a Nash point for the game is equivalent to the search of a fized point for (3.2).

The original algorithm of SCARF consists in finding a fixed point for a continuous mapping $\Gamma$ of a simplex of $\mathrm{R}^{\mathbb{N}}$ in itself. Let $\mathrm{S}$ be the simplex of $\mathrm{R}^{\mathrm{N}}$ :

$$
S=\left\{v \in R^{N} / v_{i} \geq 0 \sum_{i=1}^{N} v_{i}=1\right\} \text {. }
$$

and let us consider a partition $T_{k}$ of $S$ in sub-simplices $S_{1}, \ldots, S_{k}$. The principle of the method is to give a systematic way for walking over the vertices of the triangulation up to a vertex $u$ "which is not far from its image $\Gamma(u)$ ". More precisely the result is

Theorem 3.1 (SCARF [1.]).

Let $\varepsilon>0$ be given. Then there exists a partition $T_{k}$ of $S$ (in sub-simplices) such that the final vertex u of the algorithm satisfies.

$$
\|\Gamma(u)-u\|_{\mathrm{F}^{\mathrm{N}}} \leq \varepsilon
$$

\section{Remark 3.1}

There is an other version of this algorithn which finds a fixed point for a continuous mapping of a convex polyedron in itself but the principle of the method is the same.

\section{Remark 3.2}

There are many others algorithm to solve a fixed point problem (to say nothing of contraction mappings !), but there are all based upon the SPERNER's lemma. Hence they posiess a combinetorial aspect very sensitive to the dimensionality cf. EAVES [1], KUHH [1]。

An implementable version of the SCARF's algorithm is due to SCARF-HANSEN [2].

\section{IV - AN EXAMPLE.}

Let $Q$ be an open subset of $R^{p}, \Gamma$ its boundary, $T>0$ given and

$$
Q=\Omega \times] 0, T[, \quad \Sigma=\Gamma \times] 0, T[\text {. }
$$

We consider the following equation

(1) In the case of uncoupled constraints $(2,8)$. 
(4.1) $\left\{\begin{array}{l}\frac{d y}{d t}-\sum_{i=1}^{p} \frac{\partial}{\partial x_{i}}\left(a_{i}(x) \frac{\partial y}{\partial x_{i}}\right)=f+\sum_{j=1}^{n} v_{j}(t) \delta\left(x-c_{j}\right) \\ \left.y\right|_{\Sigma}=0 \\ y(x, 0)=y_{0}(x)\end{array}\right.$

where $c_{j}$ are given points in $\Omega$.

If we denote by $y(v)$ the unique solution of $(4.1)$ with $v=\left(v_{1}, \ldots, v_{n}\right)$ then we introduce the family of functionals :

(4.2) $\quad J_{i}(v)=\int_{0}^{T} \int_{\Omega_{i}}\left|y(x, t ; v)-z_{\dot{d}_{i}}(x, t)\right|^{2} d x d t+v_{i} \int_{0}^{T}\left|v_{i}(t)\right|^{2} d t \quad\left(v_{i} \geq 0\right)$

$\Omega_{i}$ being a subset of $\Omega$ with meas. $\left(\Omega_{i}\right) \neq 0$ for $i=1,2, \ldots$. Then if we introduce

$$
u_{a d, i}=\left\{\left.v_{i}(t)\right|_{\alpha_{i}} \leq v_{i}(t) \leq \beta_{i}\right\} \text {. }
$$

We can define our game by :

(4.3) "To find a Nash point for the family of functionals (4.2)"。

\section{Remark 4.1}

In spite of the (relative) simplicity of this problem, we cannot apply directly the theory of section 2 because of the Dirac's measures in (4.1). But this difficulty can be skipped easily by using an approximation of the $\delta$-function.

\section{Remark 4.2}

This game is an idealization of an economic problem arising in allocation of water resources between regions.

The first equation of $(4.1)$ represents the pressure of water in a phreatic. This phreatic supplies regions, each of them represented by its withdrawal :

$$
v_{j}(t) \quad \delta\left(x-c_{j}\right)
$$

where $c_{j}$ is the location of the well number $j$ and $v_{j}(t)$ is the flow-rate of water.

The cost function given by (4.2) has no direct interpretation. Actually it is necessary to modelize the economical structure of the region to exhibit the dependance of the profit with respect to the supply of water.

For a general approach of this problem cf. BREDEHOEFT-YOCNG [1].

Remark 4.3

The SCARF's algorithm is not directly applicable to problem (4.3) but it is possible to use a method analogous to the relaxation method combined with SCARF's algorithm af. YVON $[1]$ 。 


\section{REFERENCES}

A. BENSOUSSAN

$[1]$

A. BENSOUSSAN
J.L. LIONS
R. TEMAN
J.D. BREDEHOEFT
R. YOUNG
B.C. EAVES
T. HAHSEN
H. SCARF
H.W. KUHN
J.I. LIONS

$[1]$

[1]

$[1]$

[1]

[1]

[1]

[2]

J. NASH

J.B. ROSEN

H. SCARF

J.P. YVON
"Point de Nesh pour des jeux différentiels à n personnes".

To appear in SIAM J. of Control.

Cahier de l'IRIA n011. June 1972.

"The temporal allocation of a ground-water a simulation appooach".

Water Resource Research。Vol.6 n01。(1970)

"Computing Kakutani fized points"

Siam J. of Appl. Math. Vol, 21 no 2 (1971).

"On the approximation of a Nash equil.point"

Cowles Foundation. Discussion paper no272.

"Simplicial approximation of fixed points"。

Proc. NoA.S. no6 (1968).

Contrôle optimal des systèmes distribués. DUNOD (1968).

Quelaues méthodes de résolution des problèmes non Iinéaires. DUNOD Paris (1969).

[1] "Equilibrium points in $\mathbb{N}$ person game"。 Proco of $\mathrm{N}_{0} \mathrm{~A}_{\odot} \mathrm{A}$. Vol. 36 (1950).

[1] "Existence and uniqueness of Equilibrium point for concave n-person game".

Eccnometrica Vol。 33 no3 (1965).

[1] I6 "The approx。 of fixed points...".

SIAM J。 of App。 Math。 Vol。 15 n०5 (1967).

[1] To appear. 